\title{
Studies of $\mathrm{D}_{\mathrm{s}}$ decays at $B A B A R$
}

\section{Marco Pappagallo*}

Universitá and INFN, Bari, Italy

E-mail: marco.pappagallo@ba.infn.it

We present a measurement of the absolute branching fractions $\mathcal{B}\left(D_{s}^{-} \rightarrow \mu^{-} \bar{v}_{\mu}\right)$ and $\mathcal{B}\left(D_{s}^{-} \rightarrow\right.$ $\left.\tau^{-} \bar{v}_{\tau}\right)$ and of the $D_{s}$ decay constant, $f_{D_{s}}$. We also obtain an upper limit on $\mathcal{B}\left(D_{s}^{-} \rightarrow e \bar{v}_{e}\right)$. Then we present results on Dalitz plot analysis of $D_{s}^{+}$decays to $K^{+} K^{-} \pi^{+}$and $\pi^{+} \pi^{-} \pi^{+}$. The $\pi^{+} \pi^{-}$and $K^{+} K^{-} \mathcal{S}$-waves are extracted by a model-independent partial wave analysis. Finally we measure relative branching fractions of $D_{s}^{+} \rightarrow \pi^{+} \pi^{-} \pi^{+}, D_{s}^{+} \rightarrow K^{+} K^{-} K^{+}$, and $D_{s}^{+} \rightarrow K^{+} K^{+} \pi^{-}$relative to $D_{s}^{+} \rightarrow K^{+} K^{-} \pi^{+}$. All the results make use of data collected by the BABAR detector at the PEP-II storage rings at SLAC.

35th International Conference of High Energy Physics

July 22-28, 2010

Paris, France

* Speaker. 


\section{Measurement of the absolute branching fractions for $D_{s}^{-} \rightarrow \ell^{-} \bar{v}_{\ell}$ and extraction of the decay constant $f_{D_{s}}$}

The $D_{s}^{-} \rightarrow \ell^{-} \bar{v}_{\ell}$ decay ${ }^{1}$ provides a clean probe of the pseudoscalar meson decay constant $f_{D_{s}}$. Currently, the experimental values are significantly larger than theoretical predictions within the Standard Model [1]. Models of new physics (NP) may explain this difference [2].

Using a data sample of $521 \mathrm{fb}^{-1}$ [3], an inclusive sample of $D_{s}^{-}$mesons is obtained by reconstructing the rest of the event in reaction $e^{+} e^{-} \rightarrow c \bar{c} \rightarrow D K X D_{s}^{*-}$, where $D_{s}^{*-} \rightarrow D_{s}^{-} \gamma$. Here, $D$ represents a charmed hadron $\left(D^{0}, D^{+}, D^{*}\right.$, or $\left.\Lambda_{c}^{+}\right), K$ represents the $K_{S}^{0}$ or $K^{+}$, and $X$ represents additional pions produced in the $c \bar{c}$ fragmentation process. When the charmed hadron is a $\Lambda_{c}^{+}$an additional anti-proton is required to ensure baryon number conservation. No requirements are placed on the decay products of the $D_{s}^{-}$so that the selected events correspond to an inclusive sample. Having reconstructed the inclusive $D_{s}^{-}$sample, we proceed to the selection of $D_{s}^{-} \rightarrow \ell^{-} \bar{v}_{\ell}\left(\ell^{-}=e^{-}, \mu^{-}, \tau^{-} \rightarrow e^{-} \bar{v}_{e} v_{\tau} / \mu^{-} \bar{v}_{\mu} v_{\tau}\right)$ events within that sample. We require exactly one more charged particle in the remainder of the event, and that it is identified as a $e^{-} / \mu^{-}$. To extract the signal yield, we perform a binned maximum likelihood fit to the recoil mass distributions $m_{r}^{2}(D K X \gamma \ell)$ (Figs. 1 $1(\mathrm{a})$ and (b)). Since $D_{s}^{-} \rightarrow \tau^{-} \bar{v}_{\tau}$ events contain more than one neutrino, we use $E_{\text {extra }}$ to extract the yield of signal events where $E_{\text {extra }}$ is defined as the total energy of EMC clusters with individual energy greater than $30 \mathrm{MeV}$ and not overlapping with the $D K X \gamma$ candidate. Since the only missing particles in the event should be neutrinoes, we expect the distribution of $E_{\text {extra }}$ to peak at zero for signal events (Figs. I (c) and (d)).
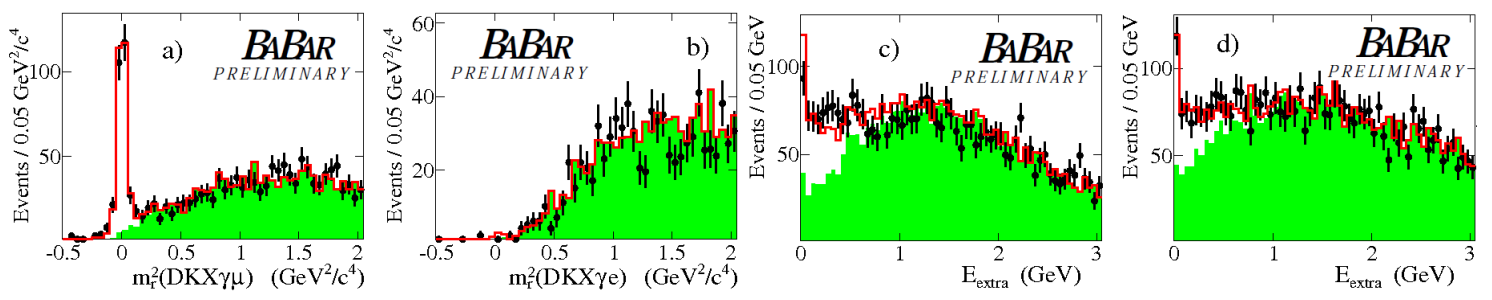

Figure 1: Fitted distributions of (a) $m_{r}^{2}(D K X \gamma \mu)$, (b) $m_{r}^{2}(D K X \gamma e)$, (c) $E_{\text {extra }}$ for $D_{s}^{-} \rightarrow \tau_{\text {ev }}^{-} \bar{v}_{\tau}$, (d) $E_{\text {extra }}$ for $D_{s}^{-} \rightarrow \tau_{\mu \nu v}^{-} \bar{v}_{\tau}$ candidates. The points represent the data with statistical error bars, the open histogram is from the fit, and the solid histogram is the background component from the fit.

The yield ratio of the above samples, corrected by the reconstruction efficiency $\varepsilon$, returns the leptonic branching fractions $\left(\mathcal{B}\left(D_{s}^{-} \rightarrow \ell^{-} \bar{v}_{\ell}\right)=\frac{N\left(D_{s}^{-} \rightarrow \ell^{-} \bar{v}_{\ell}\right)}{N\left(D_{s}^{-}\right) \varepsilon}\right)$. Then we determine the $D_{s}^{-}$decay constant for each decay mode using the known values for $m_{\ell}, m_{D_{s}},\left|V_{u d}\right|$ (we assume $\left|V_{c s}\right|=\left|V_{u d}\right|$ ), and the $D_{s}^{-}$lifetime. Finally, we obtain the error-weighted average $f_{D_{s}}=\left(258.6 \pm 6.4_{\text {stat }} \pm 7.5_{\text {syst }}\right)$ $\mathrm{MeV}$.

\section{The $D_{s}^{+}$decays and the $f_{0}(980)$ meson}

The composition of the ground state $J^{P C}=0^{++}$nonet is still unclear, and states such as the $a_{0}(980)$ or $f_{0}(980)$ may be 4 -quark states, due to their proximity to the $K \bar{K}$ threshold. This hypoth-

\footnotetext{
${ }^{1}$ All references in this paper to an explicit decay mode imply the use of the charge conjugate decay also.
} 

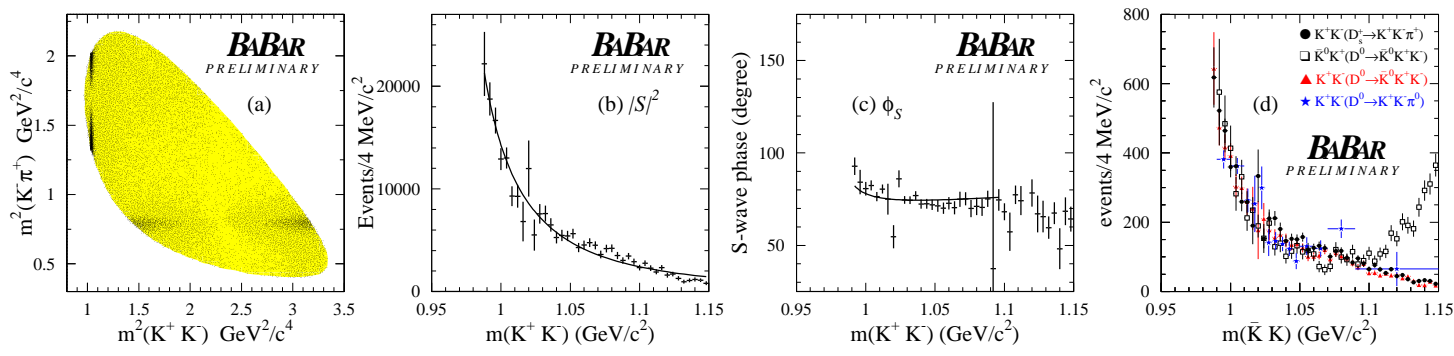

Figure 2: (a) $D_{s}^{+} \rightarrow K^{+} K^{-} \pi^{+}$Dalitz plot. (b) Squared $\mathcal{S}$-wave amplitude and (c) $\mathcal{S}$-wave phase extracted by a model independent partial wave analysis. (d) Comparison between the $K \bar{K} \mathcal{S}$-wave intensities from different charmed meson Dalitz plot analyses.

esis can be tested only through accurate measurements of the branching fractions and the couplings to different final states. In this context, $D_{s}^{+}$mesons can shed light on the structure of the $f_{0}(980)$ and the other scalar amplitudes coupled to $s \bar{s}$ [4]. In the next sections we show the results on Dalitz plot analyses of $D_{s}^{+} \rightarrow K^{+} K^{-} \pi^{+}$[5] and $D_{s}^{+} \rightarrow \pi^{+} \pi^{-} \pi^{+}$[6] using a $384 \mathrm{fb}^{-1}$ data sample.

\subsection{Dalitz plot analysis of $D_{s}^{+} \rightarrow K^{+} K^{-} \pi^{+}$}

An inclusive sample of $100,000 D_{s}^{+} \rightarrow K^{+} K^{-} \pi^{+}$events is selected by requiring the $D_{s}^{+}$meson to originate from the decay $D_{s}^{*}(2112)^{+} \rightarrow D_{s}^{+} \gamma$. The corresponding Dalitz plot is shown in Fig. 2 (a). We obtain a model-independent information on the $K^{+} K^{-} \mathcal{S}$-wave by performing a partial wave analysis in the $K^{+} K^{-}$threshold region [7]. Since the $K^{+} K^{-}$mass region is signficantly above the expected $f_{0}(980)$ mass, we observe only a threshold enhancement in the $\mathcal{S}$-wave amplitude (Fig. 2(b)) and a roughly constant trend in the $\mathcal{S}$-wave phase (Fig. $2(\mathrm{c})$ ), as would be expected for the tail of a resonance. We compare the $\mathcal{S}$-wave profile from this analysis with the $\mathcal{S}$-wave intensity values extracted from Dalitz plot analyses of $D^{0} \rightarrow \bar{K}^{0} K^{+} K^{-}$[8] and $D^{0} \rightarrow K^{+} K^{-} \pi^{0}$ [9] where an $a_{0}(980)$ resonance is also expected. We observe substantial agreement (Fig. $2(\mathrm{~d})$ ). It has been suggested that this feature supports the hypothesis that the $a_{0}(980)$ and $f_{0}(980)$ are 4-quark states [10].

An unbinned maximum likelihood fit of $D_{s}^{+} \rightarrow K^{+} K^{-} \pi^{+}$is performed in which the distribution of events in the Dalitz plot is used to determine the relative amplitudes and phases of intermediate resonant and nonresonant states. The fit results are shown in Table 1.

\begin{tabular}{lccc}
\hline Decay Mode & Decay fraction(\%) & Amplitude & \multicolumn{1}{c}{ Phase(radians) } \\
\hline \hline $\bar{K}^{*}(892)^{0} K^{+}$ & $47.9 \pm 0.5 \pm 0.5$ & 1.(Fixed) & \multicolumn{1}{c}{0. (Fixed) } \\
$\phi(1020) \pi^{+}$ & $41.4 \pm 0.8 \pm 0.5$ & $1.15 \pm 0.01 \pm 0.26$ & $2.89 \pm 0.02 \pm 0.04$ \\
$f_{0}(980) \pi^{+}$ & $16.4 \pm 0.7 \pm 2.0$ & $2.67 \pm 0.05 \pm 0.20$ & $1.56 \pm 0.02 \pm 0.09$ \\
$\bar{K}_{0}^{*}(1430)^{0} K^{+}$ & $2.4 \pm 0.3 \pm 1.0$ & $1.14 \pm 0.06 \pm 0.36$ & $2.55 \pm 0.05 \pm 0.22$ \\
$f_{0}(1710) \pi^{+}$ & $1.1 \pm 0.1 \pm 0.1$ & $0.65 \pm 0.02 \pm 0.06$ & $1.36 \pm 0.05 \pm 0.20$ \\
$f_{0}(1370) \pi^{+}$ & $1.1 \pm 0.1 \pm 0.2$ & $0.46 \pm 0.03 \pm 0.09$ & $-0.45 \pm 0.11 \pm 0.52$ \\
\hline
\end{tabular}

Table 1: Results from the $D_{s}^{+} \rightarrow K^{+} K^{-} \pi^{+}$Dalitz plot analysis. The table gives fit fractions, amplitudes and phases from the best fit. Quoted uncertainties are statistical and systematic, respectively. 

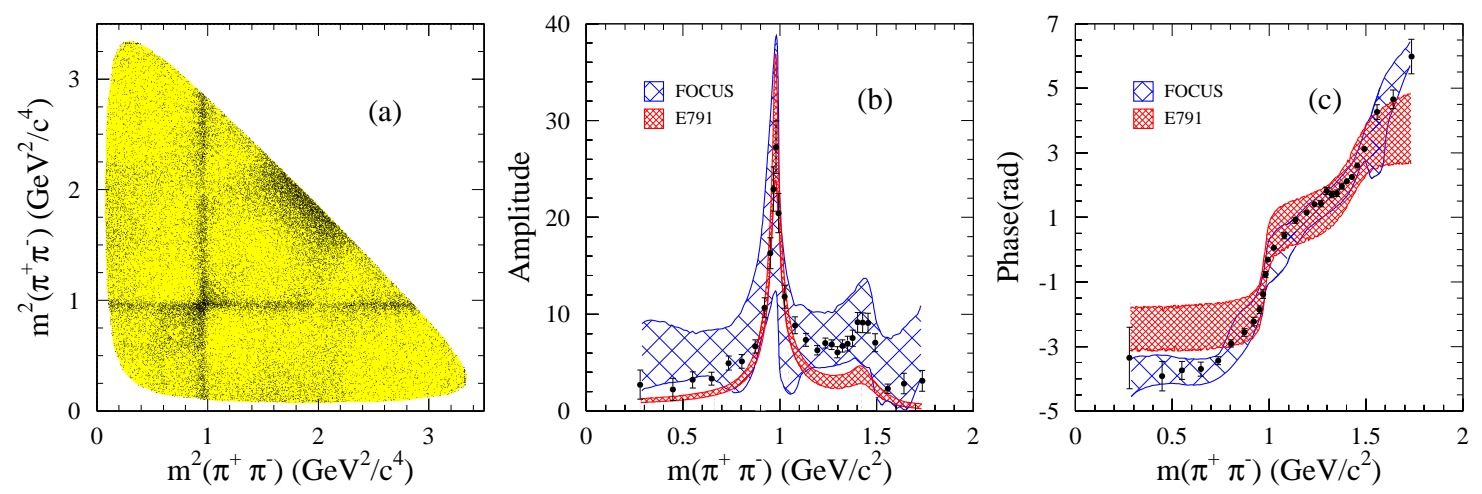

Figure 3: (a) Symmetrized $D_{s}^{+} \rightarrow \pi^{+} \pi^{-} \pi^{+}$Dalitz plot. $\pi^{+} \pi^{-} \mathcal{S}$-wave (b) amplitude and (c) phase.

We find that the decay is dominated by $\bar{K}^{*}(892)^{0} K^{+}$and $\phi(1020) \pi^{+}$amplitudes. Contributions from the $K_{1}^{*}(1410), f_{0}(1500), f_{2}(1270), \kappa(800)$ and $f_{2}^{\prime}(1525)$ are found to be consistent with zero.

\subsection{Dalitz plot analysis of $D_{s}^{+} \rightarrow \pi^{+} \pi^{-} \pi^{+}$}

An inclusive sample of $13,000 D_{s}^{+} \rightarrow \pi^{+} \pi^{-} \pi^{+}$events is reconstructed by requiring the $D_{s}^{+}$ meson to originate from the decay $D_{s}^{*}(2112)^{+} \rightarrow D_{s}^{+} \gamma$. The Dalitz plot (Fig. B(a)) shows a clear $f_{0}(980)$ signal, evidenced by the two narrow crossing bands.

An unbinned maximum likelihood fit of $D_{s}^{+} \rightarrow \pi^{+} \pi^{-} \pi^{+}$is performed on the distribution of events in the Dalitz plot to determine the relative amplitudes and phases of intermediate resonant and nonresonant states. For the $\pi^{+} \pi^{-} \mathcal{S}$-wave amplitude, we use a model-independent partial wave analysis because scalar resonances have large uncertainties. In addition, modeling the $\mathcal{S}$-wave as a superposition of Breit-Wigner functions is unphysical since it leads to a violation of unitarity when broad resonances overlap. To overcome these problems, we divide the $\pi^{+} \pi^{-}$mass spectrum into 29 slices and parametrize the $\mathcal{S}$-wave by an interpolation between the 30 end points in the complex plane. The amplitude and phase of each end point are free parameters of the fit.

The fit results (Table 1 ) show that the $D_{s}^{+} \rightarrow \pi^{+} \pi^{-} \pi^{+}$decay is dominated by the $D_{s}^{+} \rightarrow$ $\mathcal{S}$-wave $\pi^{+}$and $D_{s}^{+} \rightarrow f_{2}(1270) \pi^{+}$contributions.

\begin{tabular}{cccc}
\hline Decay Mode & Decay fraction(\%) & Amplitude & Phase(radians) \\
\hline \hline$f_{2}(1270) \pi^{+}$ & $10.1 \pm 1.5 \pm 1.0$ & .(Fixed) & 0. (Fixed) \\
$\rho(770) \pi^{+}$ & $1.8 \pm 0.5 \pm 1.0$ & $0.19 \pm 0.02 \pm 0.12$ & $1.1 \pm 0.1 \pm 0.2$ \\
$\rho(1450) \pi^{+}$ & $2.3 \pm 0.8 \pm 1.7$ & $1.2 \pm 0.3 \pm 1.0$ & $4.1 \pm 0.2 \pm 0.5$ \\
$\mathcal{S}$-wave $\pi^{+}$ & $83.0 \pm 0.9 \pm 1.9$ & Ref. [G] & Ref. [ [G] \\
\hline
\end{tabular}

Table 2: Results from the $D_{s}^{+} \rightarrow \pi^{+} \pi^{-} \pi^{+}$Dalitz plot analysis. The table reports the fit fractions, amplitudes and phases. Errors are statistical and systematic respectively.

The $\mathcal{S}$-wave shows, in both amplitude (Fig. B(b)) and phase (Fig. $3(\mathrm{c})$ ), the expected behavior for the $f_{0}(980)$ resonance and further activity is present in the regions of the $f_{0}(1370)$ and $f_{0}(1500)$ resonances. The $\mathcal{S}$-wave is small in the $f_{0}(600)$ region, indicating that this resonance has a small 
coupling to $s \bar{s}$. Figures $\bar{B}($ b) and (c) show also a comparison with the resulting $\mathcal{S}$-waves from the E791 experiment, which performed a Dalitz plot analysis using an isobar model [11], and the FOCUS experiment, which made use of the K-matrix formalism [12]. We observe that our results agree better (within uncertainties) with the results from FOCUS than those from E791.

\subsection{Measurement of the $D_{s}$ relative branching fractions}

Finally we measure the following branching ratios: $\mathcal{B}\left(D_{s}^{+} \rightarrow \pi^{+} \pi^{-} \pi^{+}\right) / \mathcal{B}\left(D_{s}^{+} \rightarrow K^{+} K^{-} \pi^{+}\right)=$ $\left(11.9 \pm 0.4_{\text {stat }} \pm 0.9_{\text {syst }}\right) \times 10^{-2}, \mathcal{B}\left(D_{s}^{+} \rightarrow K^{+} K^{+} \pi^{-}\right) / \mathcal{B}\left(D_{s}^{+} \rightarrow K^{+} K^{-} \pi^{+}\right)=\left(4.0 \pm 0.3_{\text {stat }} \pm 0.2_{\text {syst }}\right) \times$ $10^{-3}$, and $\mathcal{B}\left(D_{s}^{+} \rightarrow K^{+} K^{-} K^{+}\right) / \mathcal{B}\left(D_{s}^{+} \rightarrow K^{+} K^{-} \pi^{+}\right)=\left(2.3 \pm 0.3_{\text {stat }} \pm 0.2_{\text {syst }}\right) \times 10^{-3}$.

\section{References}

[1] E. Follana et al., High Precision Determination of the $\pi, K, D$ and $D_{s}$ Decay Constants from Lattice QCD, Phys. Rev. Lett. 100, 062002 (2008) [arXiv: 0706.1726].

[2] A. G. Akeroyd and C. H. Chen, Effect of $H^{ \pm}$on $B^{ \pm} \rightarrow \tau^{ \pm} v_{\tau}$ and $D_{s}^{ \pm} \rightarrow \mu^{ \pm} v_{\mu}, \tau^{ \pm} v_{\tau}$, Phys. Rev. D 75, 075004 (2007) [hep-ph/ 0701078 ]; B. A. Dobrescu and A. S. Kronfeld, Accumulating Evidence for Nonstandard Leptonic Decays of $D_{s}$ Mesons, Phys. Rev. Lett. 100, 241802 (2008) [arXiv: 0803.0512].

[3] P. del Amo Sanchez et al. [BaBar Collaboration], Measurement of the Absolute Branching Fractions for $D_{s}^{-} \rightarrow \ell^{-} \bar{v}_{\ell}$ and Extraction of the Decay Constant $f_{D_{s}}$, arXiv: 1008.4080.

[4] M. R. Pennington, Structure of the Scalars, arXiv: 0711.1435.

[5] P. del Amo Sanchez et al. [BaBar Collaboration], Dalitz plot analysis of $D_{s}^{+} \rightarrow K^{+} K^{-} \pi^{+}$, arXiv:1011.4190.

[6] B. Aubert et al. [BaBar Collaboration], Dalitz plot analysis of $D_{s}^{+} \rightarrow \pi^{+} \pi^{-} \pi^{+}$, Phys. Rev. D 79, 032003 (2009) [arXiv: 0808 .0971].

[7] S. U. Chung, Techniques of amplitude analysis for two-pseudoscalar systems, Phys. Rev. D 56, 7299 (1997).

[8] B. Aubert et al. [BaBar Collaboration], Dalitz plot analysis of $D^{0} \rightarrow \bar{K}^{0} K^{+} K^{-}$, Phys. Rev. D 72, 052008 (2005) [hep-ex/ 0507026$].$

[9] B. Aubert et al. [BaBar Collaboration], Amplitude analysis of the decay $D^{0} \rightarrow K^{-} K^{+} \pi^{0}$, Phys. Rev. $D$ 76, 011102 (2007) [arXiv: 0704 .3593].

[10] L. Maiani, A. D. Polosa and V. Riquer, Structure of light scalar mesons from $D_{s}$ and $D^{0}$ non-leptonic decays, Phys. Lett. B 651, 129 (2007) [hep-ph/0703272].

[11] E. M. Aitala et al. [E791 Collaboration], Study of the $D_{s}^{+} \rightarrow \pi^{-} \pi^{+} \pi^{+}$Decay and Measurement of $f_{0}$ Masses and Widths, Phys. Rev. Lett. 86, 765 (2001) [hep-ex/ 0007027$].$

[12] J. M. Link et al. [FOCUS Collaboration], Dalitz plot analysis of $D_{s}^{+}$and $D^{+}$decay to $\pi^{+} \pi^{-} \pi^{+}$using the K-matrix formalism, Phys. Lett. B 585, 200 (2004) [hep-ex/ 0312040 ]. 